\title{
Recent Advances to Estimation of Fixed-Interface Modal Models using Dynamic Substructuring
}

\author{
Mathew S. Allen \\ Department of Engineering Physics \\ University of Wisconsin \\ Madison, WI 53706 \\ msallen@engr.wisc.edu \\ \& \\ Randall L. Mayes \\ Sandia National Laboratories \\ Albuquerque, NM \\ rlmayes@sandia.gov
}

\begin{abstract}
In 2010, Allen \& Mayes proposed to estimate the fixed-interface modes of a structure by measuring the modes of the structure bolted to a fixture and then applying constraints to the fixture using the transmission simulator method. While the method proved useful, and has indeed been used in studies since that point, a few peculiarities were noted. First, in some cases the estimated fixed-base natural frequencies were observed to converge very slowly to the true values (in simulated experiments) as the number of constraints was increased. To formulate these constraints, prior studies used only the free-interface modes of the fixture or the measured modes of the assembly. This work extends that to consider other sets of constraints, showing improved results. Furthermore, in some prior studies it has been observed that there were errors of more than $10 \%$ in the natural frequencies even when the fixture motion was hundreds of times smaller than the motion of the structure of interest (and so it had presumably been removed). This work explores this phenomenon, seeking to use the strain energy in the fixture, to the extent that it can be estimated using a test-analysis model for the fixture, as a metric to predict frequency error. The proposed methods are explored by applying them to simulated measurements from a beam and from the NASA Space Launch System coupled to the Mobile Launcher.
\end{abstract}

Keywords: Component Mode Synthesis, Hurty/Craig-Bampton, Boundary Conditions, Residual Flexibility, Residual Stiffness

\section{Introduction}

The problem outlined in Fig. 1 is a variant on a classical substructuring problem, and so various methods could be used to address it. The method to be used here was developed in about 2010 and was validated through both simulations and experiments as published in [1] and as will be briefly reviewed subsequently. In most of the literature on substructuring the goal is to assemble two substructures by applying a set of constraints (enforce compatibility) and enforcing force equilibrium. The proposed application is somewhat simpler because we seek only to apply a set of constraints to one system to estimate the fixed base response. As reviewed in [2], there are two main classes of approaches: Modal Substructuring or Component Mode Synthesis (CMS) and Frequency Based Substructuring (FBS).

This paper focuses on the Modal Substructuring (CMS) family of approaches, which were pioneered by Martinez \& Carne [3], Ewins [4] and others. Early attempts proved to be extremely challenging because of the difficulty of estimating rotational responses at the interfaces; the methods are very sensitive to small errors in the measurements near the connection points. One important advance in this area occurred recently with the discovery that the substructuring could be improved by using a basis of shapes to average over several sensors near the interface [5,6] and adding additional sensors away from the interface [6,7]. In light of these findings, Allen \& Mayes developed the Transmission Simulator (TS) method [6, 8, 9] and began to report the first successful case studies in which an experimentally measured substructure was coupled to a finite element model through a continuous interface. These ideas were later used to develop a procedure to estimate the fixed-interface modes of a structure from measurements of the structure on an assembly in [1]. In that study the method was 
demonstrated on two test structures, which are shown in Fig. 1, and some results from those studies will be discussed subsequently. This is the procedure that will be used in this paper. Note, the TS is sometimes called a "Substitute" in the works of Rixen et al. [10].

The FBS family of approaches are also known by a variety of names: Impedance Coupling [11, 12], Admittance Modeling [13, 14], Receptance Coupling, Norton-Thevenin Equivalent Circuit modeling, FRF Structural Modification $[15,16]$, etc.... All of these approaches use measured frequency responses at various points on the structure (and in rare cases time domain impulse responses) to estimate the FRFs of an assembly from FRFs of each component separately. At a minimum, the input and output FRFs must be measured at each of the connection DOF, and this can lead to difficulties for multi-point connections since the FRF matrix becomes large, potentially ill-conditioned, and slight errors in reciprocity can produce anomalies in the results [13]. The challenge is understanding to what level the residual mass and stiffness from out of band modes must be captured; the anti-resonances in the FRF matrix are very sensitive to these terms. Recently, these challenges have been addressed by defining a set of basis vectors that span the space of base motion and using those to improve the conditioning in the force and response locations near the base. For example see the substructuring work done by de Klerk, Rixen et al [17, 18] and the work by Napolitano \& Yoder [19, 20]. This work focuses entirely on the modal substructuring approach, but the FBS alternative is important to keep in mind since it may prove advantageous in some situations.

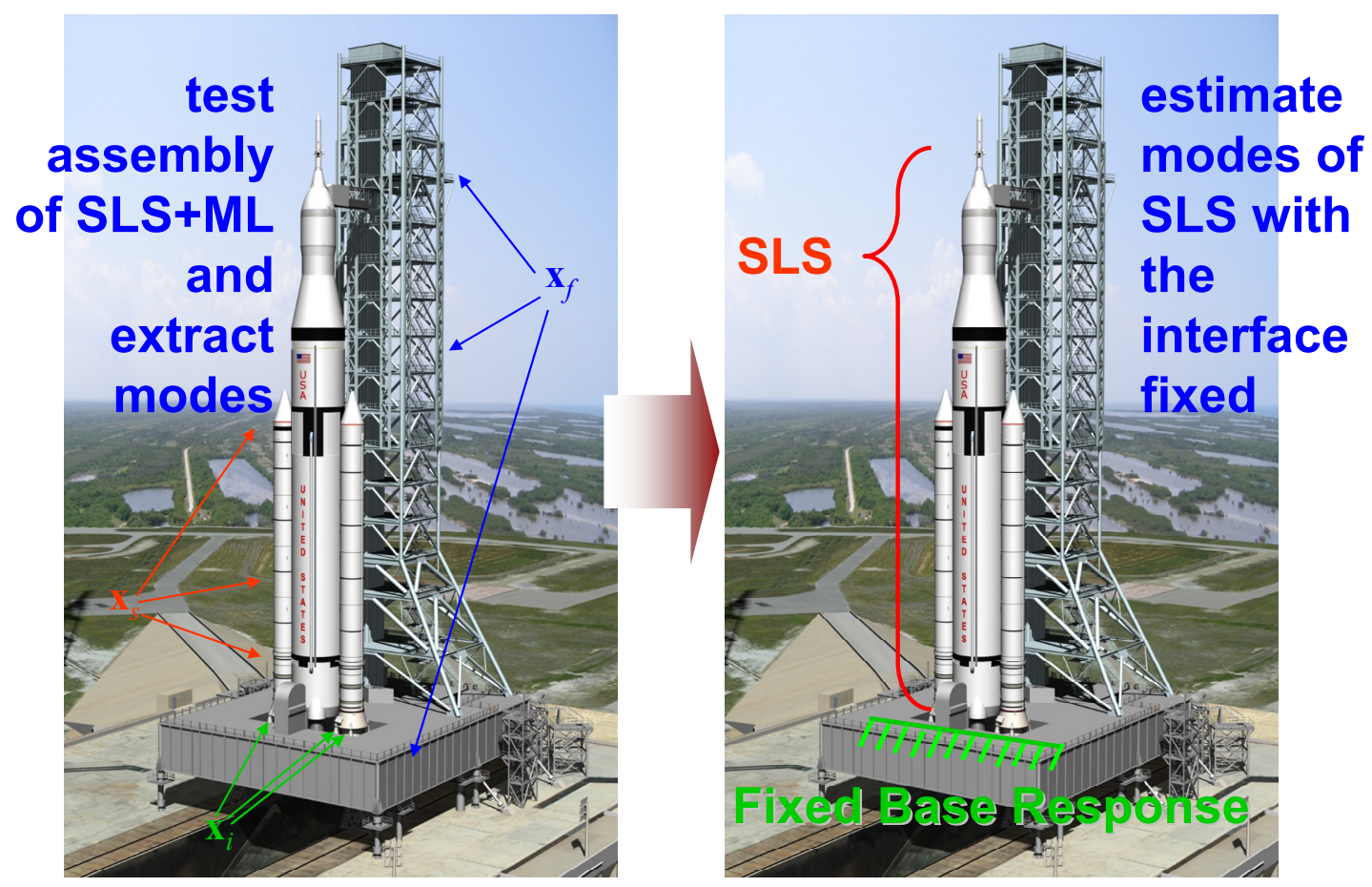

Figure 1. Schematic of one potential application of the proposed method. A modal test could be performed on the SLS when it is assembled to the ML and the modes of this assembly could be used to estimate the modes of the SLS with the interface fixed.

As explained in the abstract, this work explores two variants on the substructuring approach proposed in [1] and applies them to two structures, a simple beam and a test-analysis model of the NASA Space Launch System (SLS).

\section{Theoretical Development}

A modal test [4] can be performed on a structure to identify a linear time invariant model of a structure. The resulting modal model is comprised of $N$ modes of vibration, whose shapes are measured at a certain set of measurement points. For the purposes of this work, we presume that a test has been performed on a structure of interest which is connected to a fixture or transmission simulator [21] so the equation of motion for the fixture+structure is given by 


$$
\begin{aligned}
& {[\mathbf{I}] \ddot{\mathbf{q}}+\left[{ }^{`} 2 \omega_{r} \zeta_{r \backslash}\right] \dot{\mathbf{q}}+\left[{ }^{`} \omega_{r}{ }^{2} \backslash\right] \mathbf{q}=\left[\begin{array}{l}
\boldsymbol{\varphi}_{f} \\
\boldsymbol{\varphi}_{s}
\end{array}\right]^{\mathrm{T}}\{\mathbf{F}\}} \\
& \mathbf{x}=\left[\begin{array}{l}
\boldsymbol{\varphi}_{f} \\
\boldsymbol{\varphi}_{s}
\end{array}\right] \mathbf{q}
\end{aligned}
$$

where $\omega_{r}$ is the $r$ th natural frequency of the assembly, $\zeta_{r}$ is the corresponding damping ratio and the matrices $\boldsymbol{\varphi}_{f}$ and $\boldsymbol{\varphi}_{s}$ contain the mass-normalized mode shapes at the measurement points on the fixture, $\mathbf{x}_{f}$, and

substructure, $\mathbf{x}_{s}$, respectively.

The goal is to estimate the motion of the substructure with the interface between the fixture and substructure fixed, and this can be done if the motion of the fixture can be driven to zero. To do this, we presume that the motion of the fixture can be described by a set of shapes, $\boldsymbol{\varphi}_{f}^{\text {fixt }}$.

$$
\mathbf{x}_{f}=\varphi_{f}^{\text {fixt }} \mathbf{q}^{\text {fixt }}
$$

The following subsections will discuss various strategies for choosing the basis $\boldsymbol{\varphi}_{f}^{\text {fixt }}$.

If the fixture were truly rigid and perfectly fixed to ground, then the motion of the fixture, $\mathbf{x}_{f}$ would be zero, but all fixtures have finite stiffness, and so some motion will always be present. The most straightforward remedy would be to estimate both the displacement and rotation at the points where the structure joins the fixture and then to apply constraints to force that motion to be zero, i.e. $\mathbf{x}_{i}=0$, where $\mathbf{x}_{i}$ are the DOF at the interface (see Fig. 1). Using this approach, the number of constraints to be applied is equal to the number of interface DOF, so if the fixture+substructure has $N$ modes, and there are $N_{i}$ interface DOF, then the resulting model for the fixed-interface substructure will have $\mathrm{N}-N_{i}$ modes. While this method is straightforward, it was unfortunately illustrated in [1], that this method produces complicated results, with many spurious modes remaining in the model, and it seems to lack robustness when the measurements near the interface are noisy. Instead, in this work we prefer to use a global set of constraints applied over all DOF $\mathbf{x}_{f}$. If it is feasible to take measurements at or near the interface DOF, then those can also be included. They are not required; it is only necessary to use measurement locations which cause the fixture mode shape matrix to be easily invertible.

If the basis chosen, $\boldsymbol{\varphi}_{f}^{\text {fixt }}$, is full rank, then the fixture motions can be estimated from the measured motions, $\mathbf{x}_{f}$, as follows,

$$
\mathbf{q}^{\mathrm{fixt}} \approx\left(\boldsymbol{\varphi}_{f}^{\mathrm{fixt}}\right)^{\dagger} \mathbf{x}_{f}=\left(\boldsymbol{\varphi}_{f}^{\mathrm{fixt}}\right)^{\dagger} \boldsymbol{\varphi}_{f} \mathbf{q}
$$

where $\mathbf{q}$ denotes the modal coordinates of the assembled fixture+structure and ()$^{\dagger}$ denotes the Moore-Penrose pseudoinverse. The desired model would have negligible motion of the fixture, or in other words,

$$
\mathbf{q}^{\text {fixt }}=0 .
$$

These constraints can be applied using standard Ritz or substructuring methods $[6,8,22]$. In terms of the modal coordinates of the fixture+structure, the constraint equations are

$$
\left(\boldsymbol{\varphi}_{f}^{\mathrm{fixt}}\right)^{+} \boldsymbol{\varphi}_{f} \mathbf{q}=0,
$$

either of which constitutes a set of $N_{\text {fixt }}$ constraint equations. The matrix multiplying $\mathbf{q}$ is the matrix $[a]$ in the text by Ginsberg [22], or B in the review by De Klerk, Rixen and Voormeeren [2]. The procedure described in either of those works can be used to enforce these constraints and hence to estimate the modes of the fixture+structure 
with the fixture motion nullified. The "ritzscomb" Matlab ${ }^{\circledR}$ routine, which is freely available from the first author or on the Matlab Central File Exchange, implements the method in [22] and was used to perform the calculations for this work. For completeness, the basic operations are summarized below, using the notation from [2].

First a matrix $\mathbf{L}$ is found that resides in the null space of $\mathbf{B}=\left(\boldsymbol{\varphi}_{f}^{\mathrm{fixt}}\right)^{+} \boldsymbol{\varphi}_{f}$. Then, applying the coordinate change $\mathbf{q}=\mathbf{L} \mathbf{q}_{u}$ and pre-multipying by $\mathbf{L}^{\mathrm{T}}$ to obtain a weak form of the equations (i.e. one that ignores any forces that are not within the span of the basis), the equations of motion can be written as follows,

$$
\hat{\mathbf{M}} \ddot{\mathbf{q}}_{u}+\hat{\mathbf{C}} \dot{\mathbf{q}}_{u}+\hat{\mathbf{K}} \mathbf{q}_{u}=\mathbf{L}^{\mathrm{T}}\left[\begin{array}{ll}
\boldsymbol{\varphi}_{\mathrm{f}}{ }^{\mathrm{T}} & \boldsymbol{\varphi}_{\mathrm{s}}{ }^{\mathrm{T}}
\end{array}\right]\{\mathbf{F}\}
$$

where $\hat{\mathbf{K}}=\mathbf{L}^{\mathrm{T}}\left[{ }^{`} \omega_{r}{ }^{2} \backslash\right] \mathbf{L}$, etc...

One key challenge is to find a basis set, $\boldsymbol{\varphi}_{f}^{\text {fixt }}$, that effectively constrains the fixture to ground using the smallest possible set of shapes, in order to maximize robustness and the number of modes that can be estimated. The key method presented in [8] is reviewed below, as well as a few proposed alternatives.

\subsection{Modal Constraints (or MCFS method [8])}

Using the approach outlined in [8], a finite element model is created for the fixture, described by $\mathbf{K}^{\text {fixt }}, \mathbf{M}^{\text {fixt }}$, and then the following eigenvalue problem is solved to estimate a set of shapes, $\varphi^{\text {MCFS }}$,

$$
\left[\mathbf{K}^{f i x t}-\left(\omega^{f i x t}\right)^{2} \mathbf{M}^{f i x t}\right] \boldsymbol{\varphi}^{\mathrm{MCFS}}=0
$$

which are partitioned to the measurement DOF and used as the desired basis, i.e. $\boldsymbol{\varphi}_{f}^{\mathrm{fixt}}=\boldsymbol{\varphi}_{f}^{\mathrm{MCFS}}$.

In the study in [8], the authors applied this approach to a plate with a beam attached, as shown in Fig. 2. In that study, the authors found that even after constraining out many mode of the free-free plate, the resulting mode shapes of the cantilever beam agreed very well with theory, yet the natural frequencies estimated were in error by between 3 and 10\%. The study was repeated with the experimental measurements replaced with modes from a FEM (to eliminate measurement errors) and the natural frequencies had almost the same pattern of errors. This revealed that modal truncation was the culprit, i.e. that it was not possible to completely nullify the motion of the fixture due to an incomplete basis for the fixture+substructure and an incomplete set of shapes $\boldsymbol{\varphi}_{f}^{\text {fixt }}$. A further study using the FEM revealed that the natural frequency errors decreased to between 0.2 and $3 \%$ if the plate was made three times thicker near the connection point (schematic on the right in Fig. 2). 

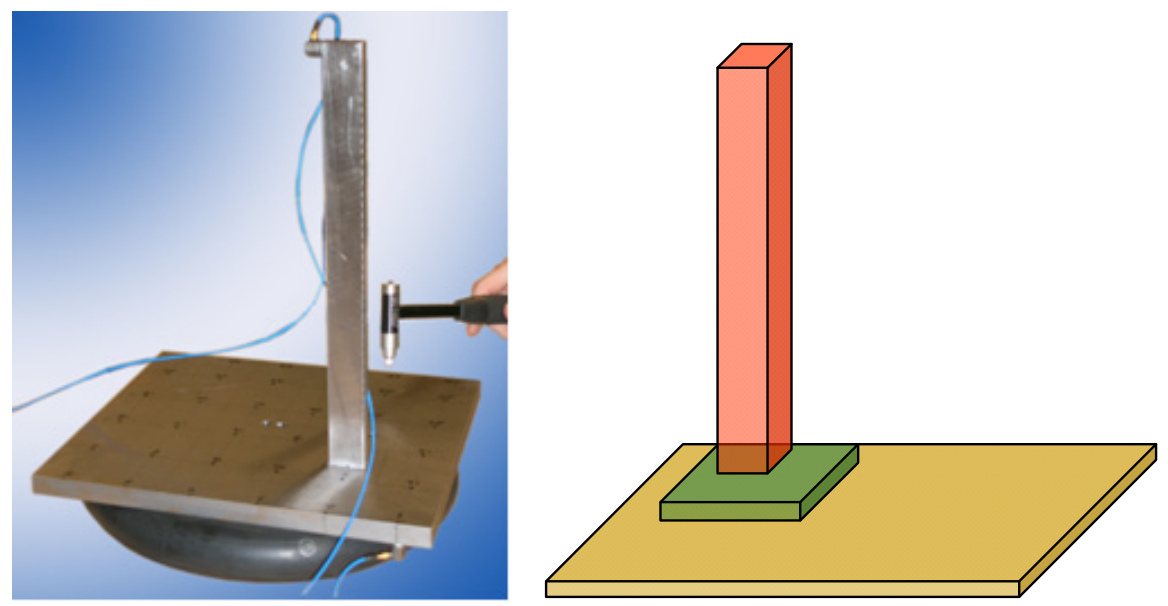

Figure 2. (left) Photograph of a 0.5 " thick by 1.0 " wide steel beam mounted on a 0.625 " thick steel plate. The modes of this system were measured at a grid of points on the plate and used to estimate the fixed-interface modes of the beam. (right)

Schematic of a FEM of the structure with the region near the interface made thicker (more rigid).

\subsection{Modal Constraints with Rigidized Substructure (MC-R)}

In a recent work, it was noted that MCFS shapes don't exercise the interface, and hence this might explain the slow convergence of the MCFS approach that was seen in [8]. If the mass properties of the substructure are known, then a rigid mass can readily be added to the finite element model of the fixture to load the interface and hopefully produce a better basis. The resulting FEM is denoted $\mathbf{K}^{f i x t+R}, \mathbf{M}^{f i x t+R}$, and once again an eigenvalue problem is solved to estimate the motion of the fixture+rigid substructure.

$$
\left[\mathbf{K}^{f i x t+R}-\left(\omega^{f i x t+R}\right)^{2} \mathbf{M}^{f i x t+R}\right] \boldsymbol{\varphi}^{\mathrm{MCR}}=0
$$

which are partitioned to the measurement DOF and used as the desired basis, i.e. $\boldsymbol{\varphi}_{f}^{\mathrm{fixt}}=\boldsymbol{\varphi}_{f}^{\mathrm{MCR}}$.

It is important to note that the constraints in Eqs. (7) and (8) only enforce zero motion at the fixture measurement points if the number of measurement points equals the number of fixture modes, but ideally one would actually use more measurement points on the fixture than there are active modes in the structure in order to average out noise and measurement errors. As a result, the motions of the physical measurement points will typically not be exactly zero after applying the constraints. In the best case the residual motion would be due only to measurement noise, but experience has shown that there is typically some residual motion in the fixture that is physical, since one is seeking to constrain an infinite dimensional system with a finite number of constraints. Fortunately, one can readily observe the fixture motions after applying the constraints to see whether the constraints were effective in enforcing a rigid boundary condition, and this was demonstrated in [8]. In this work we take this a step further, seeking to use the FEM of the fixture and the measured fixture motions to obtain better estimates of the natural frequencies of the substructure on a rigid foundation.

\subsection{Natural Frequency Correction Scheme}

In the example cited in Fig. 2, even after using the best available set of constraints, the natural frequencies estimated continued to show errors. It was noted that even though the residual motions of the fixture (the plate in Fig. 2) were small, less than $1 \%$ of the motion of the structure of interest (the vertical beam), because the stiffness of the plate is very high, it is possible that exceedingly small motions of the fixture have an important contribution to the strain energy in each mode, and hence to the natural frequencies.

In this work, we propose to use Test Analysis Models (TAMs) derived from finite element models of the fixture to estimate the error in the natural frequencies due to the residual flexibility that remains in the fixture. Consider an undamped structure that is vibrating in the $r$ th mode, so that $\mathbf{x}(t)=\operatorname{Re}\left(\boldsymbol{\varphi}_{r} e^{i \omega_{r} t}\right)$. The peak potential and kinetic energies must be equal, and the kinetic energy, $K E_{\text {true }}=\frac{1}{2} \dot{\mathbf{x}}^{\mathrm{T}} \mathbf{M} \dot{\mathbf{x}}$ can then be rewritten to find that the 
maximum kinetic energy is $\max (K E)=\omega_{r}{ }^{2} \times \frac{1}{2} \boldsymbol{\varphi}_{r}{ }^{\mathrm{T}} \mathbf{M} \boldsymbol{\varphi}_{r}$. Similarly, the maximum potential energy is $\max (P E)=\frac{1}{2} \boldsymbol{\varphi}_{r}{ }^{\mathrm{T}} \mathbf{K} \boldsymbol{\varphi}_{r}$, and equating these two we obtain the following equation that relates the $r$ th natural frequency to the energy in the structure.

$$
\omega_{r}{ }^{2}=\frac{P E}{K E^{*}}=\frac{\frac{1}{2} \boldsymbol{\varphi}_{r}{ }^{\mathrm{T}} \mathbf{K} \boldsymbol{\varphi}_{r}}{\frac{1}{2} \boldsymbol{\varphi}_{r}{ }^{\mathrm{T}} \mathbf{M} \boldsymbol{\varphi}_{r}},
$$

where the notation $K E^{*}$ reminds the reader that this is not the true kinetic energy, but an energy like quantity.

The equation above can be expanded in a Taylor series to obtain the desired relationship,

$$
2 \omega_{r} \frac{\partial \omega_{r}}{\partial P E}=\frac{1}{K E^{*}}
$$

where we have assumed that the kinetic energy does not change significantly. This is in line with our experience, where the mode shape of the structure as a whole is nearly unchanged, so that the kinetic energy would not change significantly. The potential energy can then be decomposed by separating the mode shape into DOF on the structure of interest, denoted $\boldsymbol{\varphi}_{S r}$, and those on the fixture or transmission simulator, $\boldsymbol{\varphi}_{F r}$. We have measurements of the natural frequency and mode shape for a case where the fixture motion is non-negligible, $\left.\omega_{r}\right|_{\text {meas }}$ and we desire to know the natural frequency that would be obtained if the fixture motion were zero $\left.\omega_{r}\right|_{0}$. Expanding about the latter case, the relationship becomes the following.

$$
\left.\omega_{r}\right|_{\text {meas }}=\left.\omega_{r}\right|_{0}+\frac{1}{2 \omega_{r} K E^{*}}\left(P E_{S}-P E_{S, 0}+P E_{F}-P E_{F, 0}+P E_{J}-P E_{J, 0}\right)
$$

where

$$
P E_{S}=\frac{1}{2} \boldsymbol{\varphi}_{S r, \text { meas }}{ }^{\mathrm{T}} \mathbf{K} \boldsymbol{\varphi}_{S r, \text { meas }},
$$

and so forth for the other sensor sets and for the measured case, ()|meas, and the case where the fixture motion is zero $\left.()\right|_{0}$.

Before proceeding it is helpful to illustrate using a simple example. Consider a spring attached to ground via two springs in series, $k_{s}$ representing the stiffness of the system of interest and $k_{f}$ representing the stiffness of a fixture. Then let the mass and $k_{s}$ be unity as the stiffness of the fixture is varied between 1 and 100 (i.e. $1 \mathrm{x}$ to $100 \mathrm{x}$ stiffer than the system of interest). The natural frequency of the system was then computed and is shown in Fig. 3 . The line labeled "Measured" corresponds to the natural frequency that would be measured on the elastic fixture, while the line labeled "True" is the frequency that we would obtain if the fixture was infinitely stiff. The measured frequency can be seen to decrease as the stiffness of the boundary decreases. The regime of interest are those cases where the natural frequency shows a small (i.e. $\sim 10 \%$ ) error. 

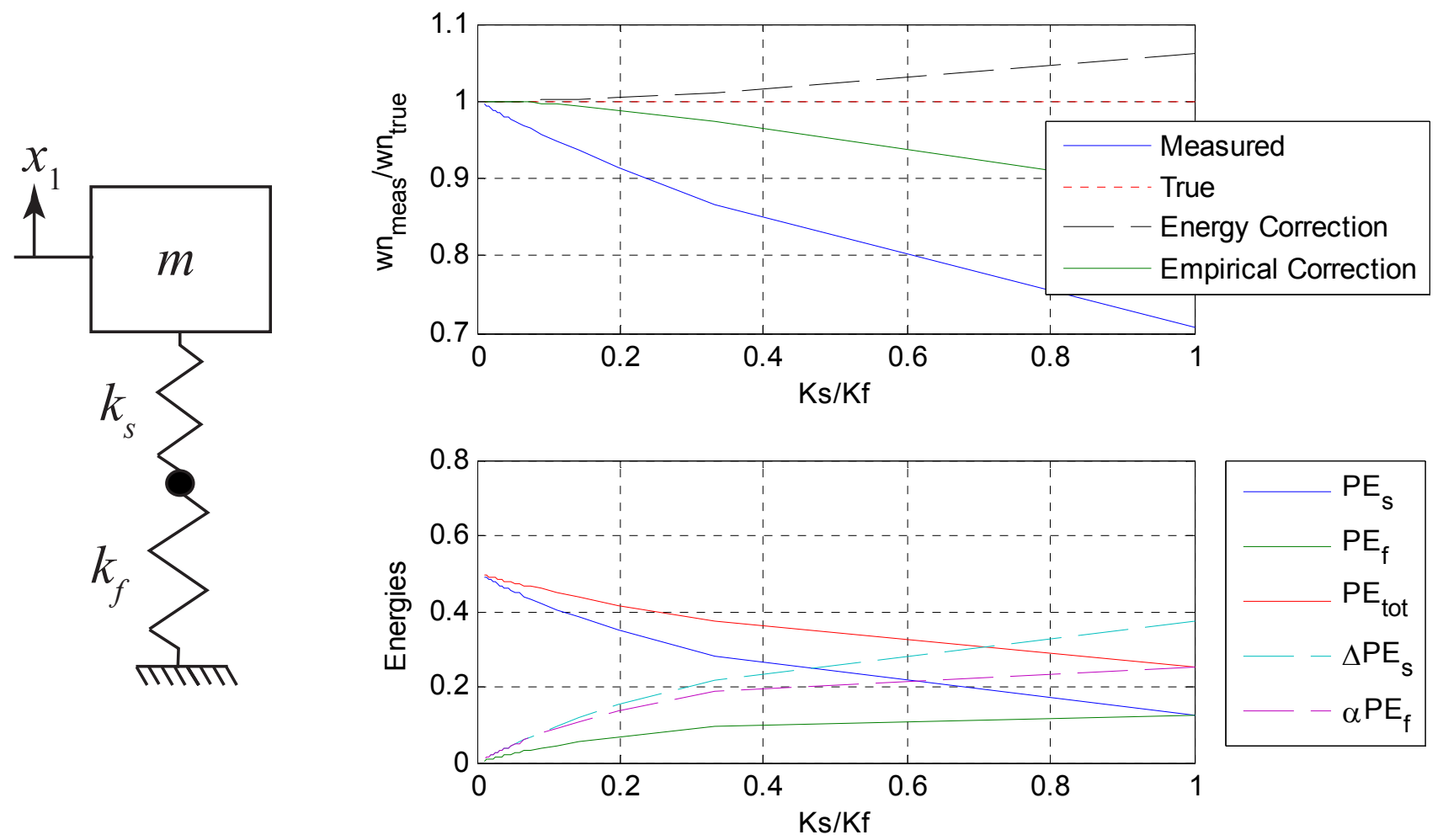

Figure 3. (a) Ratio between the true natural frequency (i.e. that measured on an infinitely stiff foundation) and that measured on a foundation with stiffness $\underline{k}_{f}$. (b) The potential energy (PE) in each of the springs $\underline{k}_{\underline{s}}$ and $\underline{k}_{f}$ as a function of the ratio $k_{s} / k_{f}$.

For the magenta curve, $\alpha=2.0$.

The second pane in Fig. 3 shows the energy in each of the springs as a function of their relative stiffness. As the fixture becomes stiffer (with $\mathrm{Ks} / \mathrm{Kf}$ approaching 0 ), less and less energy is stored in the fixture and the energy in the system approaches that which it would have on a rigid foundation. If the energies $\triangle P E_{S}=P E_{S}-P E_{S, 0}$ and $\triangle P E_{F}=P E_{F}-P E_{F, 0}=P E_{F}-0$ were known, then one could use Eq. (11) to correct the measured natural frequency, as shown by the dashed black line in Fig. 3a. Unfortunately, while we expect that $\triangle P E_{F}$ could be estimated using a high quality ROM of the fixture or transmission simulator, it seems less likely that $\triangle P E_{S}$ could be reliably estimated.

It appears that the only viable option is to use some empirical means to estimate the other energy quantities from $\triangle P E_{F}$. For example, in the simple case shown, $\triangle P E_{S}$ is approximately equal to $-\alpha P E_{F}$, with $\alpha=2.0$, as shown with the magenta line in Fig. 3. Using this assumption, the empirical natural frequency correction scheme becomes the following

$$
\left.\omega_{r}\right|_{\text {meas }}=\left.\omega_{r}\right|_{0}+\frac{1}{2 \omega_{r} K E^{*}}\left((1-\alpha) \frac{1}{2} \boldsymbol{\varphi}_{F r, \text { meas }}{ }^{\mathrm{T}} \mathbf{K} \boldsymbol{\varphi}_{F r, \text { meas }}\right),
$$

and the result for this simple system, shown in Fig. $3 \mathrm{a}$, can be seen to work quite well up to $\underline{k}_{s} / k_{f}=0.2$, or to the point where the error in the natural frequency is about $10 \%$. In the absence of other information, we shall presume that this empirical factor, $\alpha=2.0$, is reasonable for structures in general and evaluate the method for other structures. 


\section{Numerical Results}

These two concepts, using mass-loaded shapes to enhance the constraint basis (Section 2.2), and the natural frequency correction scheme (Section 2.3), were tested on two structures to evaluate their performance. The results are discussed in the following subsections.

\subsection{Free-Free Beam}

The first structure considered is an assembly of two free-free beams, shown below. The beam on the left is the "fixture" or transmission simulator, and the goal is to estimate the modes of the beam on the right when it is perfectly fixed. Two cases are simulated using one-dimensional finite element models. For all of the cases the beams have the following properties: height, $\mathrm{h}=0.75 \mathrm{in}$, width, $\mathrm{b}=1 \mathrm{in}$, Young's Modulus, $\mathrm{E}=30.0 \mathrm{e} 6 \mathrm{psi}$, density, $\rho=0.28 \mathrm{lb} / \mathrm{in}^{\wedge} 3$. For Case 1 , the lengths of the beams are $\mathrm{L}_{\mathrm{A}}=12 \mathrm{in}, \mathrm{L}_{\mathrm{B}}=24 \mathrm{in}$, while Case 2 represents a more challenging situation in which Beam $A$ is much more flexible relative to Beam $B$, with $L_{A}=20$ in, $L_{B}=16$ in. For all of the cases considered, we use all modes of A and B up to $6400 \mathrm{~Hz}$, in other words we presume that the modal test on each component is able to extract all of the modes from 0 to $6400 \mathrm{~Hz}$, including the rigid body modes.
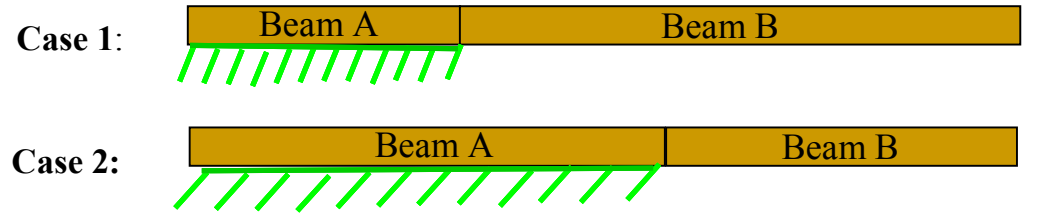

Figure 4. Schematic of the beam system studied in this section. Two cases will be considered, Case 1 (top) and Case 2 (bottom), which differ in the relative lengths of Beams A and B.

The first step is to compute the mode shapes of the transmission simulator when a rigid mass is used in place of the structure of interest. Figure 5 shows the resulting mode shapes for Case 1; these shapes are used in Eq. (5) to eliminate the motion of the transmission simulator. One can see that the rigid mass enriches the modal basis, as the resulting shapes show asymmetry and involve deformation near the interface.

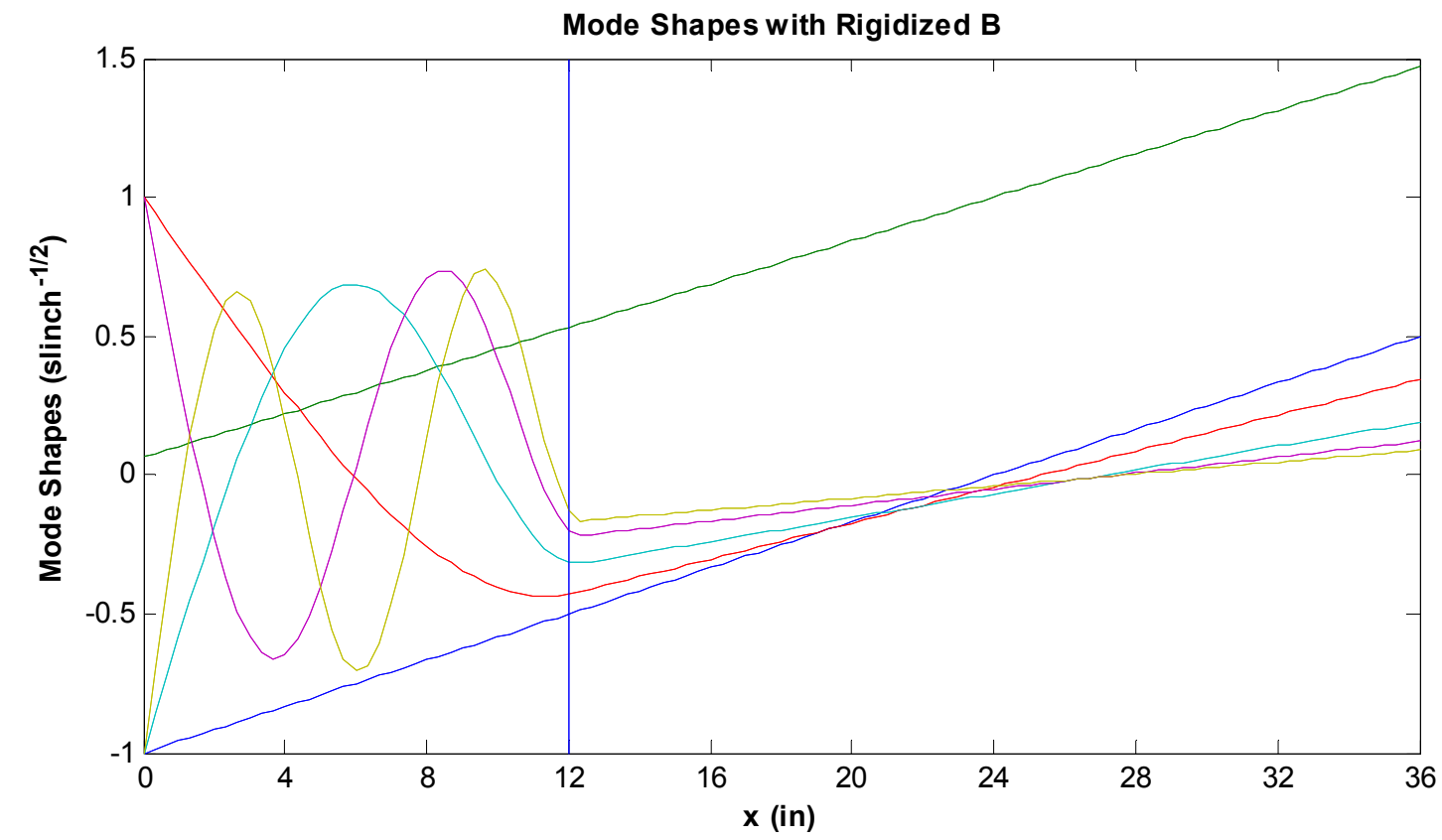

Figure 5. Case 1: Mode shapes of the Transmission Simulator (Beam A) when Beam B is replaced by a rigid mass. This is the basis of shapes used to form the constraints in Eq. (5).

The standard modal substructuring approach is then used to enforce these constraints, and the natural frequencies and mode shapes of the system with Beam A fixed are then computed. For Case 1, the resulting shapes are 
compared with the true fixed-interface shapes in Fig. 6 and the natural frequencies are compared in Table 1. For this case there were 12 modes of the assembly and 5 constraint shapes below $6400 \mathrm{~Hz}$, and so this modal basis produces estimates for the first 7 modes of beam B. A Test-Analysis-Model (TAM) was created of Beams A and $\mathrm{B}$ using the displacement of each node (the rotations were statically reduced out), and this was used to estimate the potential energy in Beam A when it deformed into each mode, and the kinetic energy of Beam B.
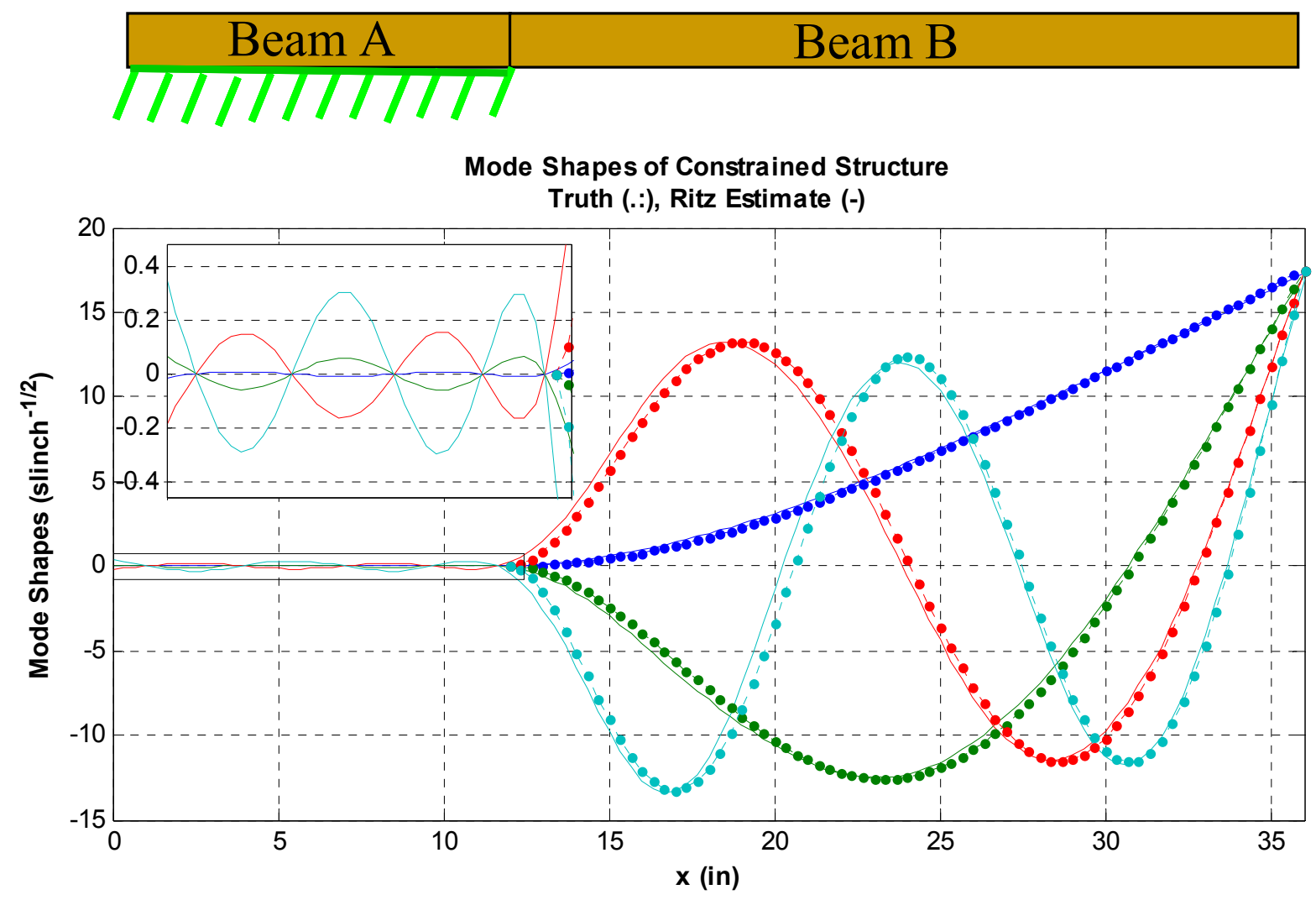

Figure 6. Case 1: Mode shapes of Beam B with the Fixed-Interface fixed. Solid lines show the true fixed-interface shapes of the FEM, while the dotted lines show those estimated by Modal Substructuring using the MC-R method in Sec. 2.2.

Table 1: Case 1: Natural Frequencies estimated by substructuring for two cases: ( $A=$ Free-Free) when the modes of the transmission simulator are computed with the interface free, (A+Rigid B) when the system of interest is replaced with a rigid mass, and (A+Rigid B, Corrected) the latter case with the frequencies corrected using the empirical formula.

\begin{tabular}{|c|c|c|c|c|c|c|}
\hline $\begin{array}{l}\text { B Freq. } \\
\text { True }\end{array}$ & $\begin{array}{l}\text { B Freq. } \\
\text { CMS } \\
\text { A=Free- } \\
\text { Free }\end{array}$ & $\%$ Error & $\begin{array}{c}\text { B Freq. } \\
\text { CMS } \\
\text { A+Rigid } \\
\text { B }\end{array}$ & $\%$ Error & $\begin{array}{c}\text { B Freq. } \\
\text { CMS } \\
\text { A+Rigid B, } \\
\text { Corrected }\end{array}$ & $\%$ Error \\
\hline 42.8 & 39.9 & -6.7 & 40.7 & -4.9 & 41.8 & -2.3 \\
\hline 268.2 & 251.5 & -6.2 & 256.1 & -4.5 & 262.1 & -2.3 \\
\hline 751.0 & 707.5 & -5.8 & 720.1 & -4.1 & 738.7 & -1.6 \\
\hline 1471.6 & 1393.2 & -5.3 & 1417.2 & -3.7 & 1464.9 & -0.5 \\
\hline 2432.7 & 2314.7 & -4.9 & 2353.6 & -3.3 & 2456.9 & 1.0 \\
\hline 3634.1 & 3478.3 & -4.3 & 3534.5 & -2.7 & 3723.9 & 2.5 \\
\hline 5075.7 & 4903.9 & -3.4 & 4975.5 & -2.0 & 5255.9 & 3.6 \\
\hline
\end{tabular}


The results show that adding a Beam B to the transmission simulator enriches the basis, reducing the errors in the natural frequencies noticeably, although not dramatically. When using a free-free transmission simulator, the error, show in the third column, range between -3 and $-7 \%$ In contrast, when a rigid Beam B is used to enrich the basis, the errors drop to -2 to $5 \%$. The empirical natural frequency correction scheme improves the results further, most notably reducing the error in the first natural frequency from $-4.9 \%$ to $-2.3 \%$. However, for some of the higher natural frequencies the scheme over-corrects the frequencies. When considering this, one should note that when using substructuring, they typical rule of thumb states that the natural frequencies should be accurate up to $50-66 \%$ of the frequency range used for each subcomponent, or 3200 to $4300 \mathrm{~Hz}$ in this case. However, here we obtain excellent results up to $80 \%$ of the frequency range. In our experience, if the fixture is quite rigid (i.e. well approximated as a rigid mass) then the results may be excellent in the entire frequency range. In contrast, if the fixture is very flexible then the results may be poor even below $50 \%$ of the measured frequency range.

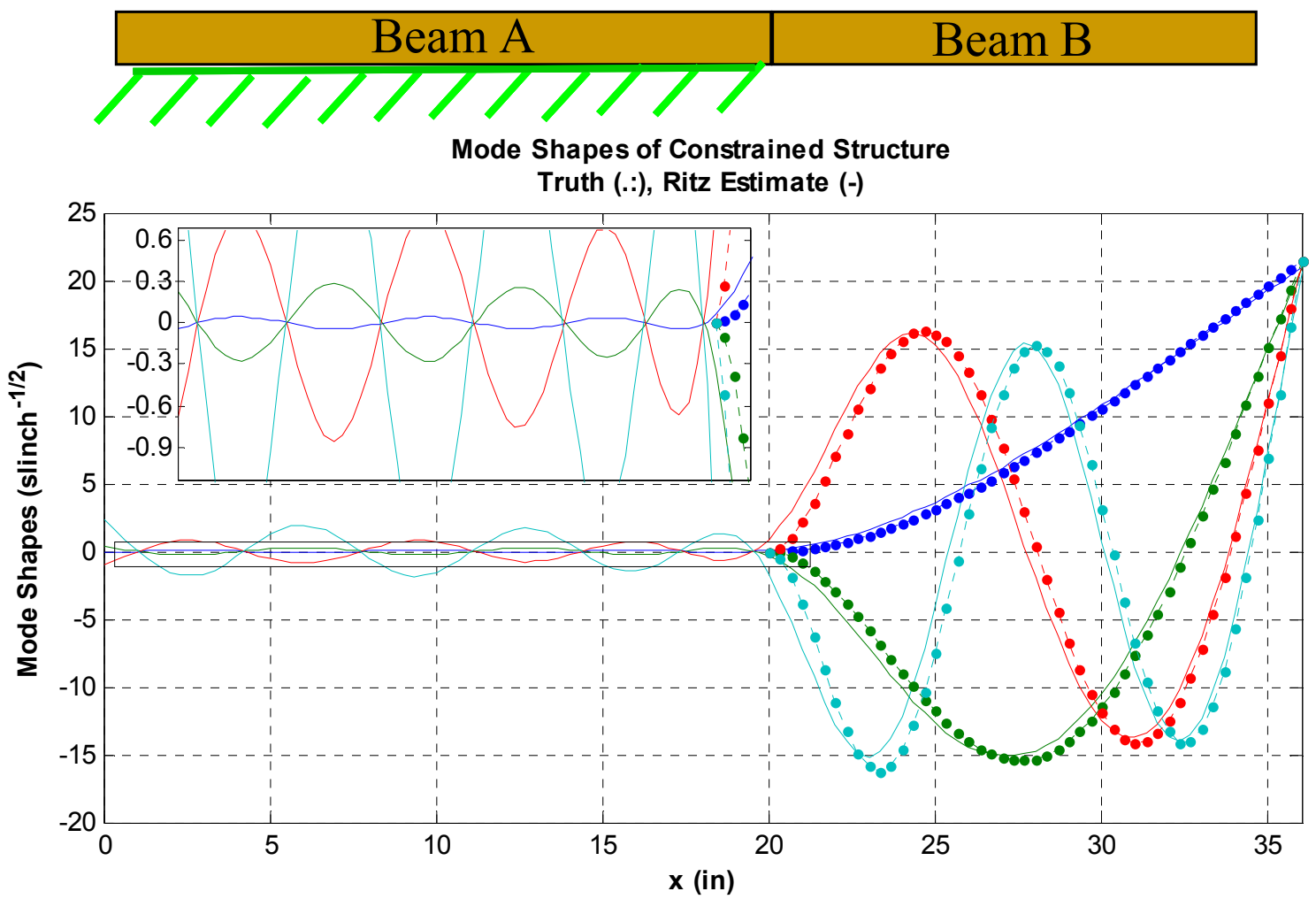

Figure 7. Case 2: Mode shapes of Beam B with the Fixed-Interface fixed. Solid lines show the true fixed-interface shapes of the FEM, while the dotted lines show those estimated by Modal Substructuring using the MC-R method in Sec. 2.2.

Next consider the case where Beam A is longer, i.e. the fixture is more flexible relative to the structure of interest. In this case there are once again 12 modes of the assembly below $6400 \mathrm{~Hz}$, but now we have 7 constraint shapes in that frequency range. As might be expected, the more flexible fixture produces errors in the natural frequencies that are much larger, ranging between -11 and $-13 \%$. In this case using the enhanced basis for Beam A reduces these errors to -7 to $-9 \%$. Table 2 also shows the natural frequencies corrected using the empirical scheme with $\alpha=2$. While the correction scheme works very well for the first mode, it increases the errors for the other modes, dramatically for the highest frequency modes. In the case of those modes, it may be that the strain energy is so large that the Taylor series expansion is no longer valid. 
Table 2: Case 2: Natural Frequencies estimated by substructuring for two cases: ( $A=$ Free-Free) when the modes of the transmission simulator are computed with the interface free, (A+Rigid B) when the system of interest is replaced with a rigid mass, and (A+Rigid $\mathrm{B}$, Corrected) the frequencies corrected using the empirical formula.

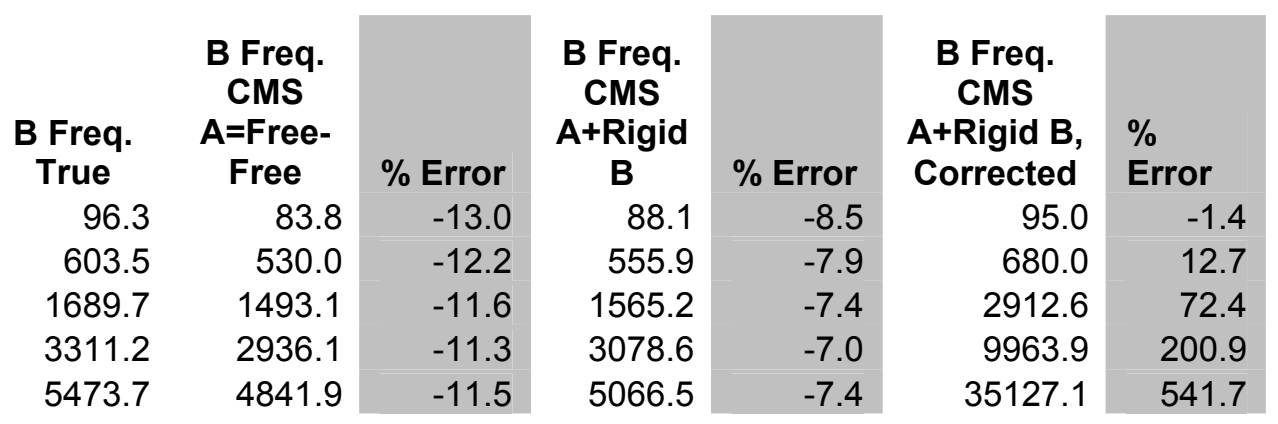

\subsection{NASA Space Launch System on Mobile Launcher}

The methods were further evaluated by testing them on the new Space Launch System (SLS) that NASA is developing. While legacy vehicles, such as the Saturn V and the Space Shuttle, were tested in free-free conditions in order to update the corresponding finite element models, NASA no longer has a facility with the capability to lift and hang even the initial 70t payload model so that it could be tested free free. This initial model is expected to weigh approximately 5.5 million pounds, or the equivalent of 7.5 fully-loaded 747 jets ${ }^{1}$. Instead, plans are being developed to test the SLS while it is mounted on the Mobile Launch (ML) platform, within the Vehicle Assembly Building (VAB). This test is internally called the Integrated Modal Test (IMT). Even though the SLS is more massive than the SLS, the ML has significant low frequency dynamics, so it will become necessary to also model the ML in order to correlate to this test. This is a significant undertaking, since the ML is a complicated frame structure with thousands of bolted structural members, extensive cabling systems, etc...

One possible alternative would be to use the measured modes of the SLS assembled to the ML (SLS+ML) in order to predict the modes that the SLS would have on a truly rigid foundation (i.e. a fixed interface). This section uses test analysis models of the SLS and ML to explore this possibility. Statically reduced test-analysis models (TAMs) were provided with a few thousand degrees of freedom, and which were used to estimate the motion of the (SLS+ML) at 625 measurement DOF. The measurement DOF were chosen to distinguish between the (SLS+ML) modes, as measured by the MAC and Cross-Orthogonality. The model also contains 24DOF at the interface between the SLS and ML (note that the restraining arm towards the top of the tower was not connected to the SLS). These DOF consist of translations at 8 posts, four at the base of each Solid Rocket Booster (SRB). These 24DOF were fixed in the model to estimate the true fixed based modes of the SLS, and that model will be used as a truth model.

Two case studies will be presented here. In the first the TAMs will be used to estimate the free-free modes of the ML, and the same fixed-interface method that was presented in [1] is used, which is here denoted ML-F since the ML has a free-interface. For the second case, the modes of the ML will be computed with the SLS replaced with a rigid mass, as proposed in Sec. 2.2, and the results will be denoted ML-R. In both cases, we presume that the first 149 modes of the SLS+ML are extracted from the modal test, corresponding to all modes in a certain frequency range. Then 84 modes of the ML-F or ML-R are used as constraints, corresponding to all modes of the ML in approximately the same frequency range. The ML-F actually had about $10 \%$ more modes in the frequency range of interest, but the same number of constraints was used to obtain a more straightforward comparison.

Table 3 shows the errors in the natural frequencies and the corresponding MAC values for three cases. First, it is interesting to consider the errors that would be obtained if the SLS+ML modes were compared directly with the modes of the SLS-Fixed. If this is done, the natural frequencies have errors ranging between -12.4 and $1.4 \%$ over the first ten modes, and the MAC values range between 0.92 and 1.00, so the ML does form a fairly effective fixed-interface for the SLS. However, it is important to note that SLS+ML contains many additional modes and

\footnotetext{
${ }^{1}$ https://www.nasa.gov/pdf/588413main_SLS_Fun_Facts.pdf, accessed October 2017.
} 
this table only shows those SLS+ML modes that best matched the true SLS-Fixed modes. The modes estimated by CMS reduce these frequency errors, to between $-6.0 \%$ and $0.6 \%$ for the ML-F method and $-11.0 \%$ and $-0.1 \%$ for the ML-R method. The improvement in the MAC between the modes is even more significant, with most MAC values above 0.97 . However, for a few of the modes the MAC values decrease significantly, most notably to 0.87 for the $4^{\text {th }}$ mode in the ML-R results.

Table 3: Errors in the natural frequencies estimated by substructuring for the SLS. Shaded modes are the primary modes of interest.

\begin{tabular}{|c|c|c|c|c|c|c|c|c|c|}
\hline \multirow[b]{2}{*}{$\begin{array}{l}\text { SLS } \\
\text { Mode \# }\end{array}$} & \multicolumn{2}{|c|}{$\begin{array}{l}\text { Measured } \\
\text { SLS+ML }\end{array}$} & \multicolumn{3}{|c|}{$\begin{array}{l}84 \text { Constraints: Modes of } \\
\text { ML-R (i.e. SLS Rigidized) }\end{array}$} & \multirow{2}{*}{$\begin{array}{l}\text { ML-R } \\
\text { Corrected } \\
\text { Freq. \% } \\
\text { Error }\end{array}$} & \multicolumn{3}{|c|}{$\begin{array}{l}84 \text { Constraints: Modes of } \\
\text { ML-F (i.e. SLS Removed) }\end{array}$} \\
\hline & $\begin{array}{l}\text { Freq. \% } \\
\text { Error }\end{array}$ & MAC & $\begin{array}{l}\text { CMS } \\
\text { Mode \# }\end{array}$ & $\begin{array}{l}\text { Freq. \% } \\
\text { Error }\end{array}$ & MAC & & $\begin{array}{l}\text { CMS } \\
\text { Mode \# }\end{array}$ & $\begin{array}{l}\text { Freq. \% } \\
\text { Error }\end{array}$ & MAC \\
\hline 1 & -12.4 & 1.00 & 1 & -11.0 & 1.00 & 2.0 & 1 & -2.6 & 1.00 \\
\hline 2 & -9.4 & 1.00 & 2 & -5.1 & 1.00 & -3.2 & 2 & -6.0 & 1.00 \\
\hline 3 & -8.7 & 0.92 & 4 & -1.7 & 0.98 & -1.3 & 4 & 0.3 & 1.00 \\
\hline 4 & -6.8 & 0.95 & 3 & -6.0 & 0.87 & -4.0 & 3 & -5.9 & 0.99 \\
\hline 5 & -2.1 & 0.95 & 5 & -0.5 & 1.00 & -0.5 & 5 & -0.7 & 1.00 \\
\hline 6 & -3.4 & 1.00 & 6 & -2.6 & 0.98 & -2.5 & 6 & -3.3 & 0.99 \\
\hline 7 & 1.4 & 0.99 & 7 & -2.9 & 0.98 & -0.6 & 7 & 0.5 & 0.93 \\
\hline 8 & -1.6 & 0.94 & 8 & -3.3 & 0.97 & -2.9 & 8 & -3.2 & 0.79 \\
\hline 9 & -2.2 & 0.97 & 9 & -2.3 & 0.98 & -0.6 & 9 & 0.6 & 0.96 \\
\hline 10 & -1.9 & 0.95 & 10 & -0.1 & 1.00 & 0.0 & 10 & -0.4 & 1.00 \\
\hline 11 & -2.1 & 0.97 & 11 & -2.2 & 0.99 & -0.4 & 11 & -1.9 & 0.99 \\
\hline 12 & -2.2 & 0.99 & 12 & -2.8 & 0.97 & -2.4 & 12 & -2.3 & 0.95 \\
\hline 13 & -6.3 & 0.98 & 13 & -5.4 & 0.82 & -2.8 & 13 & -5.8 & 0.95 \\
\hline 14 & -1.6 & 0.92 & 14 & -1.8 & 0.87 & 8.5 & 14 & -2.1 & 0.96 \\
\hline 15 & -6.5 & 0.76 & 15 & -1.1 & 0.64 & 18.8 & 15 & -1.1 & 0.70 \\
\hline 16 & -35.2 & 0.45 & 16 & -1.2 & 0.80 & 5.5 & 16 & 6.3 & 0.43 \\
\hline 17 & -0.1 & 0.99 & 18 & 0.3 & 0.98 & 22.9 & 18 & -0.3 & 0.98 \\
\hline 18 & 0.8 & 0.39 & 20 & -0.5 & 0.32 & 6.0 & 20 & -1.2 & 0.27 \\
\hline 19 & -1.5 & 0.87 & 19 & -4.8 & 0.67 & -0.8 & 19 & -9.0 & 0.43 \\
\hline 20 & -6.9 & 0.70 & 17 & -8.7 & 0.13 & 9.7 & 17 & -7.7 & 0.24 \\
\hline
\end{tabular}

Figure 8 shows the shapes of the second mode of the fixed-interface SLS for two cases: the corresponding mode of the SLS+ML (Mode 6) and the second mode estimated by substructuring using ML-R constraints. The character of the modes is difficult to discern from these static plots, but they do show that the ML base and tower show significant motion for the SLS+ML mode, even if Table 3 shows that its frequency and shape are fairly accurate. In contrast, the modes estimated from substructuring show no visible motion of the ML tower or base. 

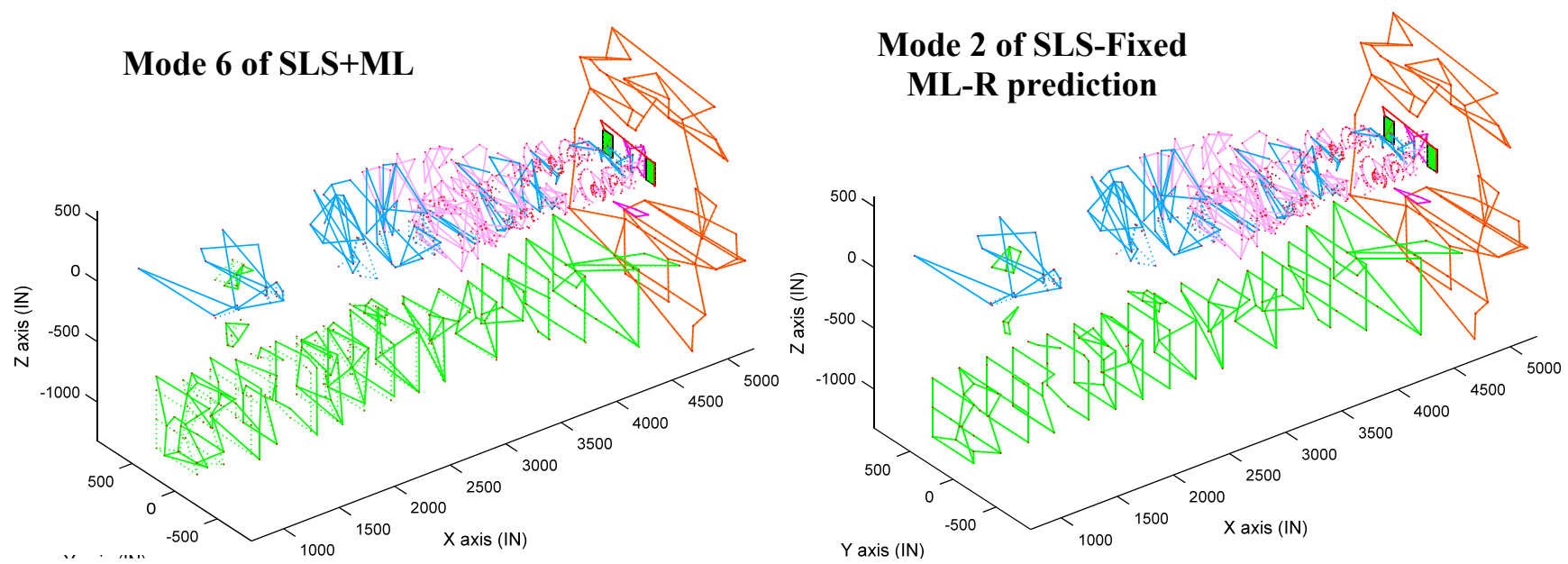

Figure 8. Mode shapes of Mode 2 from (left) the measurements on the SLS+ML, i.e. the first column in Table 3, and (right) from substructuring using the ML rigidized, i.e. ML-R.

Figure 9 shows the MAC between the modes estimated by substructuring, with the true modes of the SLS with a fixed interface (SLS-Fixed). The MAC plots show that in both ML-F and ML-R cases there is a good correspondence between the true modes and those estimated by substructuring. However, the ML-R results show better agreement for the higher frequency modes, while the ML-F results seem to still contain several spurious modes beyond about the $16^{\text {th }}$.
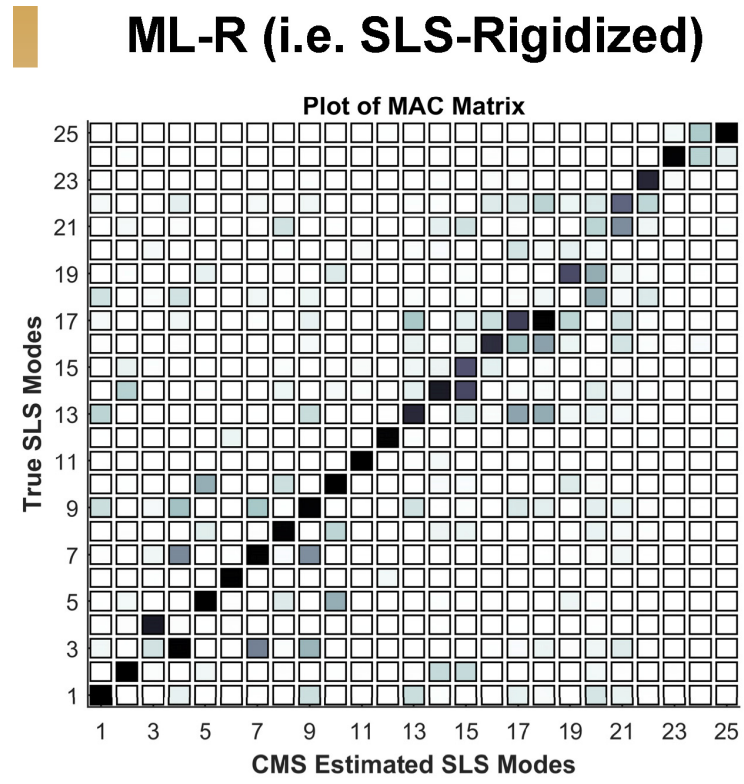

\section{ML-F (i.e. w/out SLS)}

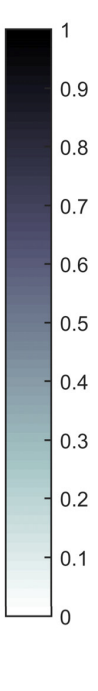

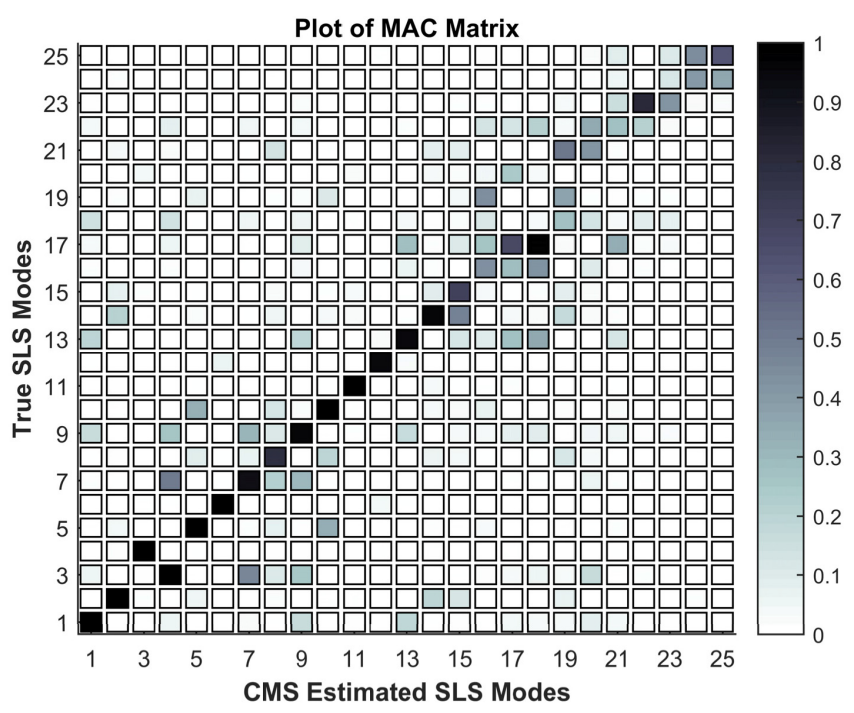

Figure 9. MAC comparing the modes of the SLS-Fixed as estimated using substructuring with those of the truth model for two cases: (ML-F, right) the ML with a free interface, (ML-R, left) the ML with a rigid mass connected to the interface.

It is also informative to consider the drive point FRFs at a few points as would be obtained by the various models. Drive points near the top of the SLS were used that were approximately in the axial and the two lateral directions, and the FRFs were reconstructed using the modes from the ML-F and ML-R method and are shown in Fig. 10. The FRF show that the ML-R method is far superior in estimating the high frequency axial modes (middle row), although it is less accurate for the first bending mode in the in-out direction and for the higher frequency in-out modes. 


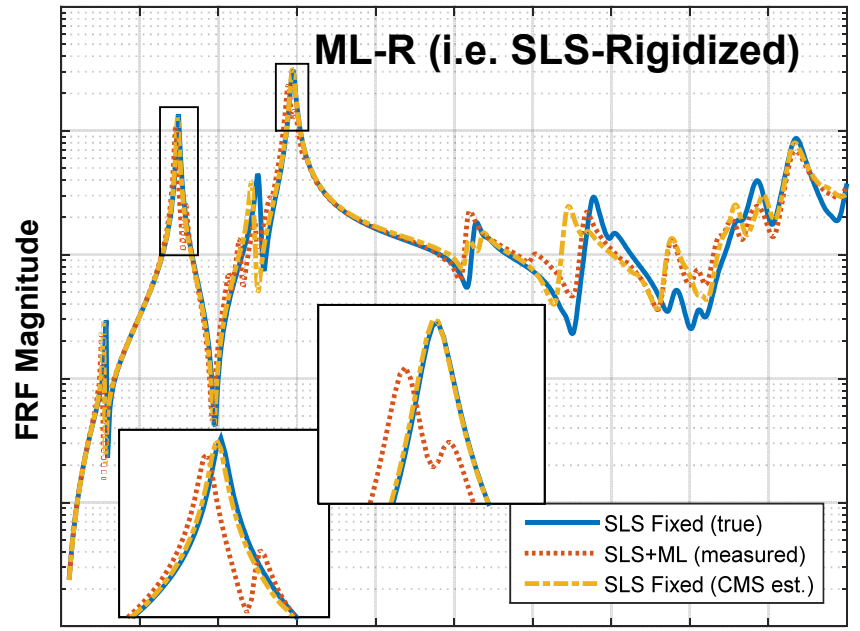

Frequency

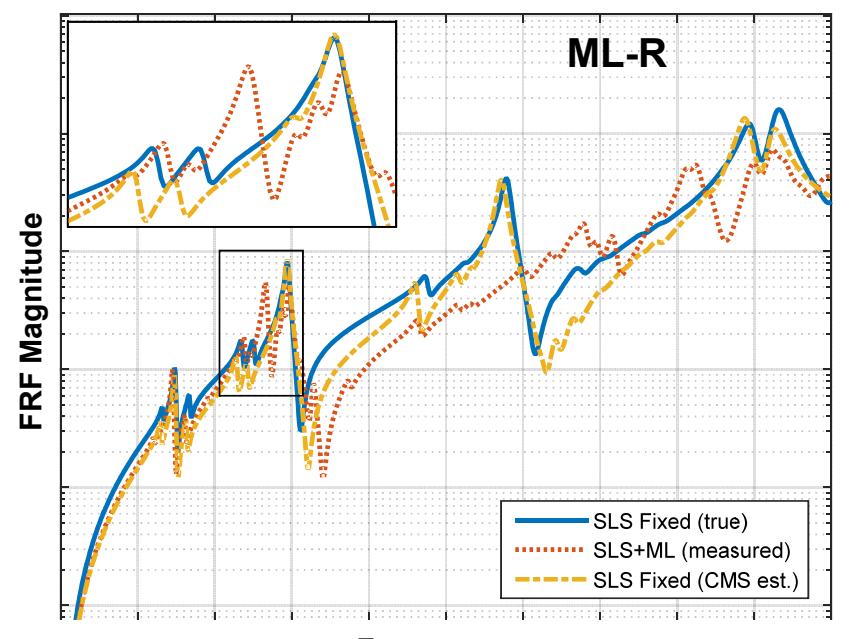

Frequency

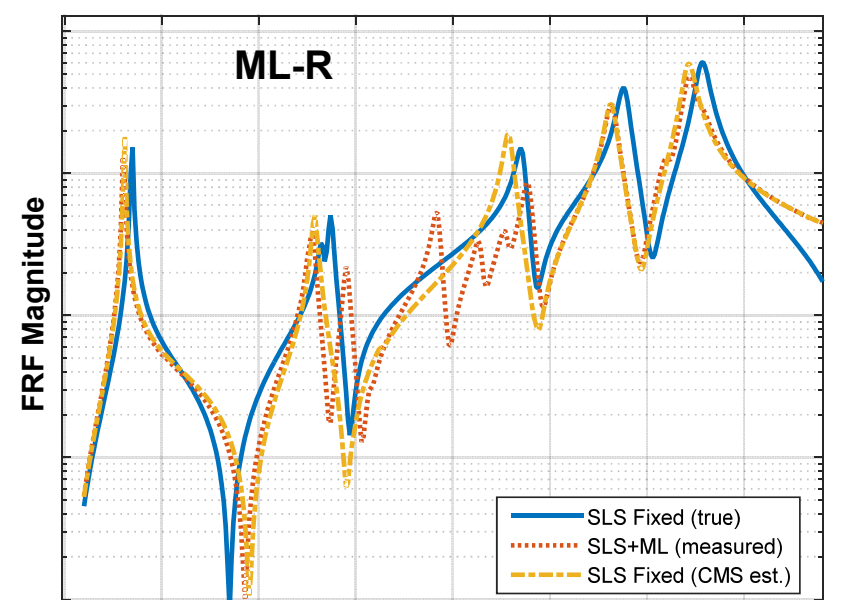

Frequency

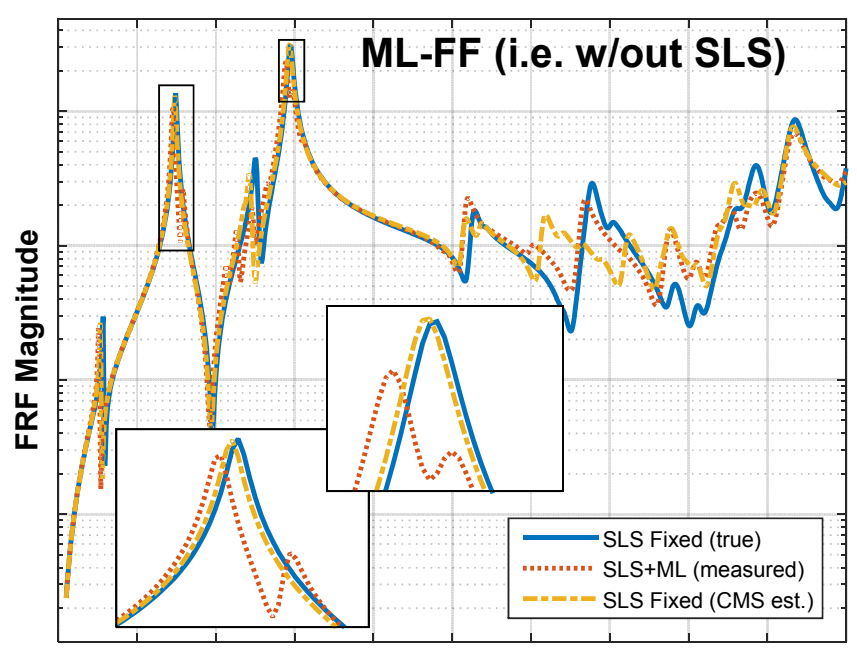

Frequency

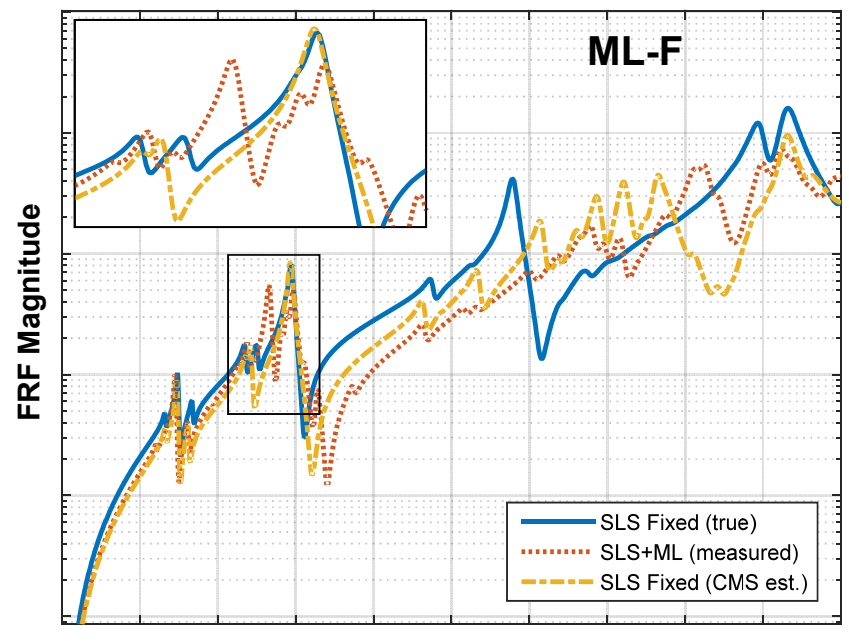

Frequency

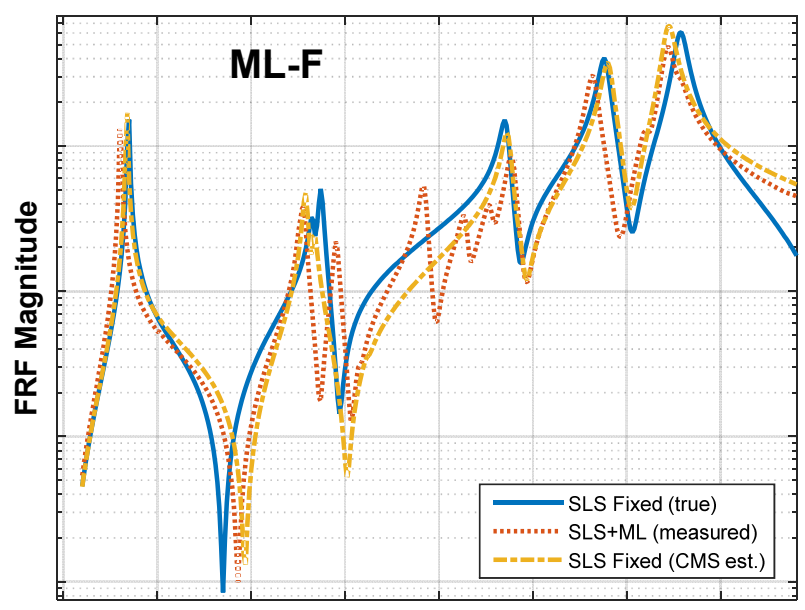

Frequency

Figure 10. Drive-point frequency response functions for the SLS-Fixed as estimated using substructuring with those of the truth model for two cases: (ML-F, right) and (ML-R, left). The FRFs are also shown for the SLS+ML, or the FRFs that would be obtained in the test of the coupled system if no post processing is performed. (top) - Lateral direction, (middle) Axial direction, (bottom) - Bending towards/away from ML tower 


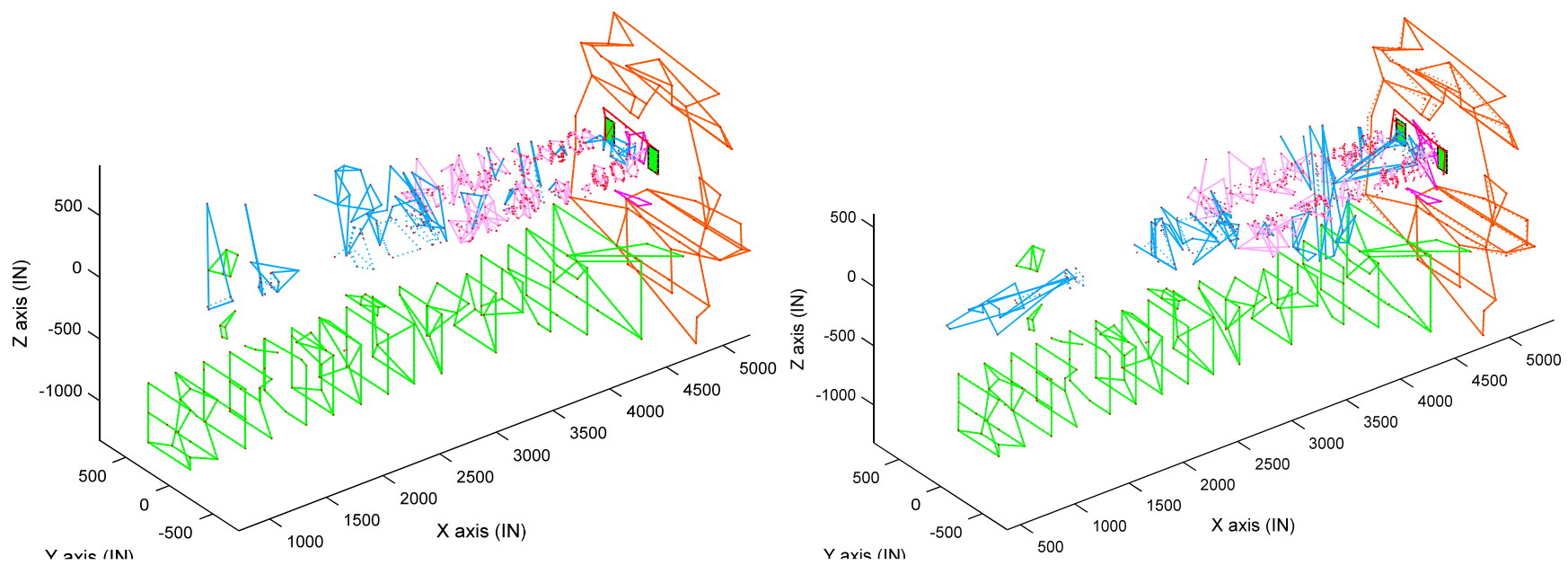

Figure 11. Mode shapes of Modes 1 (left) and 16 (right) for the ML-R case. The character of the modes is difficult to discern from these static plots, but they do show that the ML base and tower shows no visible motion for Mode 1, while it shows some residual vibration for the axial Mode 16.

As was done in the previous section, we can visualize the residual motion in the fixture (i.e. the ML) to understand how effective the constraints were. Figure 11 shows the SLS+ML for two modes estimated using the ML-R approach. The residual motion in the ML is not visible, but it is still presumed to be the cause of the frequency errors seen in Table 3. In order to evaluate this, the natural frequency correction scheme was evaluated for this system using $\alpha=2$ as in the previous section and using the mode shapes estimated by the ML-R method. The method proves effective at correcting the natural frequencies of the first few modes, reducing the errors in those frequencies from between $-11.0 \%$ to $-0.5 \%$ to $-4 \%$ to $2.0 \%$.

\section{Conclusions}

Two enhancements have been presented to the method proposed by Allen, Gindlin \& Mayes [1] that estimates the fixed-interface modes of a structure that is connected to a flexible fixture. The first is a small modification to the modal basis used to define the constraints that are applied to the fixture; local deformations near the interface are captured by adding a rigid mass to the fixture before computing its modes. This was found to improve the estimates of the structure's fixed-interface natural frequencies to a certain extent, although not dramatically. We also explored the use of schemes to correct the natural frequencies estimated for the fixed-interface structure, based on the strain energy remaining in the fixture, as estimated by a Test-Analysis Model. The theoretical development revealed that, because the change in Potential Energy in the structure of interest could not be estimated accurately, it was necessary to assume that it was proportional to the energy in the fixture, and this makes the method empirical and calls its general applicability into question. However, for the cases studied to date the empirical method seemed to provide good results so long as the deformation in the fixture was not too large. A general statement that continues to hold with these and other investigations since the publication of [1] is that the fewer modes the fixture has in the measured bandwidth, the better the substructuring approach works to estimate the fixed base result.

\section{Acknowledgements}

This research is based on work that was originally supported by Sandia National Laboratories. Sandia National Laboratories is a multi-mission laboratory managed and operated by National Technology and Engineering Solutions of Sandia, LLC., a wholly owned subsidiary of Honeywell International, Inc., for the U.S. Department of Energy's National Nuclear Security Administration under contract DE-NA-0003525. The authors also gratefully acknowledge the support of the National Aeronautics and Space Administration (NASA) and the helpful comments provided by members of the Loads and Dynamics Technical Discipline Team of the NASA Engineering \& Safety Center (NESC). 


\section{References}

[1] M. S. Allen, H. M. Gindlin, and R. L. Mayes, Experimental Modal Substructuring to Estimate Fixed-Base Modes from Tests on a Flexible Fixture. Journal of Sound and Vibration 330 (2011) 4413-4428.

[2] D. de Klerk, D. J. Rixen, and S. N. Voormeeren, General framework for dynamic substructuring: History, review, and classification of techniques. AIAA Journal 46 (2008) 1169-1181.

[3] D. R. Martinez, T. G. Carne, D. L. Gregory, and A. K. Miller, Combined Experimental/Analytical Modeling Using Component Mode Synthesis, AIAA/ASME/ASCE/AHS Structures, Structural Dynamics \& Materials Conference, Palm Springs, CA, USA, 1984.

[4] D. J. Ewins, Modal Testing: Theory, Practice and Application, Research Studies Press Baldock, England, 2000.

[5] M. Corus, E. Balmes, and O. Nicolas, Using model reduction and data expansion techniques to improve SDM. Mechanical Systems and Signal Processing 20 (2006) 1067-89.

[6] M. S. Allen and R. L. Mayes, Comparison of FRF and Modal Methods for Combining Experimental and Analytical Substructures, 25th International Modal Analysis Conference (IMAC XXV), Orlando, Florida, 2007.

[7] P. Sjovall and T. Abrahamsson, Substructure system identification from coupled system test data. Mechanical Systems and Signal Processing 22 (2008) 15-33.

[8] M. S. Allen, R. L. Mayes, and E. J. Bergman, Experimental Modal Substructuring to Couple and Uncouple Substructures with Flexible Fixtures and Multi-point Connections. Journal of Sound and Vibration 329 (2010) 4891-4906.

[9] R. L. Mayes, P. S. Hunter, T. W. Simmermacher, and M. S. Allen, Combining Experimental and Analytical Substructures with Multiple Connections, 26th International Modal Analysis Conference (IMAC XXVI), Orlando, Florida, 2008.

[10] D. de Klerk and D. J. Rixen, Component transfer path analysis method with compensation for test bench dynamics. Mechanical Systems and Signal Processing 24 (2010) 1693-1710.

[11] A. P. V. Urgueira, Dynamic Analysis of Coupled Structures Using Experimental Data, PhD Thesis, Imperial College of Science, Technology and Medicine, London: University of London, 1989.

[12] M. Imregun, D. A. Robb, and D. J. Ewins, Structural Modification and Coupling Dynamic Analysis Using Measured FRF Data, 5th International Modal Analysis Conference (IMAC V), London, England, 1987.

[13] T. G. Carne and C. R. Dohrmann, Improving Experimental Frequency Response Function Matrices for Admittance Modeling, 24th International Modal Analysis Conference (IMAC XXIV), St. Louis, Missouri, 2006.

[14] D. R. Martinez, A. K. Miller, and T. G. Carne, Combined Experimental and Analytical Modeling of Shell/Payload Structures, The Joint ASCE/ASME Mechanics Conference, Albuquerque, NM, 1985.

[15] J. E. Mottershead, A. Kyprianou, and H. Ouyang, Structural modification. Part 1: rotational receptances. Journal of Sound and Vibration 284 (2005) 249-65.

[16] A. Kyprianou, J. E. Mottershead, and H. Ouyang, Structural modification. Part 2: Assignment of natural frequencies and antiresonances by an added beam. Journal of Sound and Vibration 284 (2005) 267-281.

[17] D. De Klerk, Dynamic Response Characterization of Complex Systems Through Operational Identification and Dynamic Substructuring, PhD Thesis: Delft University of Technology, 2009.

[18] D. de Klerk, D. J. Rixen, S. N. Voormeeren, and F. Pasteuning, Solving the RDoF Problem in Experimental Dynamic Substructuring, 26th International Modal Analysis Conference (IMAC XXVI), Orlando, Florida, 2008.

[19] K. Napolitano and N. Yoder, Fixed Base FRF Using Boundary Measurements as References - Analytical Derivation, 30th International Modal Analysis Conference (IMAC XXX), Jacksonville, FL, 2012.

[20] N. Yoder and K. Napolitano, Fixed Base FRF Using Boundary Measurements as References Experimental Results, 30th International Modal Analysis Conference (IMAC XXX), Jacksonville, FL, 2012.

[21] R. L. Mayes and M. Arviso, Design Studies for the Transmission Simulator Method of Experimental Dynamic Substructuring, International Seminar on Modal Analysis (ISMA2010), Lueven, Belgium, 2010.

[22] J. H. Ginsberg, Mechanical and Structural Vibrations, First ed., John Wiley and Sons New York, 2001. 